\title{
Medical Image of the Week: Intraventricular Hemorrhage Casting
}

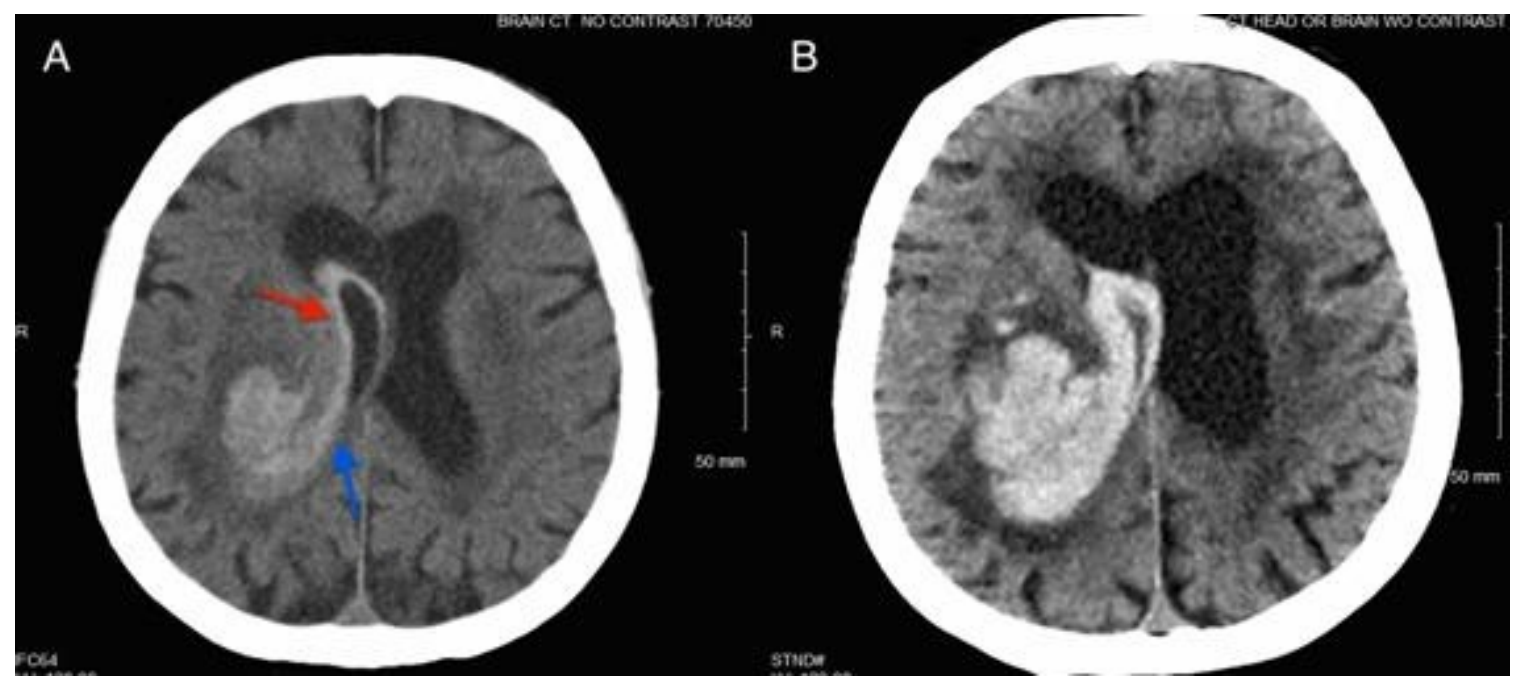

Figure 1. Panel A: Computerized tomography of the head without contrast taken at an outlying facility displayed a right thalamic intraparenchymal hematoma, measuring $3.4 \times 4.2 \mathrm{~cm}$, with vasogenic edema and intraventricular rupture (blue arrow). Intraventricular hemorrhage casting is visualized in the right lateral ventricular causing obstructive hydrocephalus (red arrow). Panel B: Repeat noncontrast CT of the head 6 hours later revealed an increase in size of thalamic

hematoma to $4.3 \times 5.2 \times 4.8 \mathrm{~cm}$, an increase in amount of Intraventricular hemorrhage, progression of hydrocephalus from cast obstruction, and worsening vasogenic edema causing $5 \mathrm{~mm}$ left midline shift.

An 80-year-old woman with a past medical history of hypertension and hypercholesterolemia presented to an outlying hospital at 11:00 hours with slurred speech, left arm drift, and headache. A non-contrast CT of the head revealed an intraparenchymal hematoma in the right thalamus measuring $3.4 \mathrm{x}$ $4.2 \mathrm{~cm}$ with an associated intraventricular rupture (Figure 1A, blue arrow). An intraventricular hemorrhage cast with secondary hydrocephalus was also noted on initial imaging (Figure 1A, red arrow). She was placed on a nicardipine drip for blood pressure control and subsequently transferred to OSF St. Francis Medical Center (OSFMC) for a higher level of care.

Upon arrival to OSFMC, the patient was poorly responsive, non-verbal, and could not follow commands. She was directly admitted to the Neuroscience Intensive Care Unit for further management. Vitals signs were stable on presentation. Neurologic examination revealed a comatose patient with asymmetric and minimally reactive pupils, absent gag reflex, right gaze preference, brisk corneal reflex on the right and absent response on the left, absent deep tendon reflexes on the left upper and lower extremity, with absent response to painful stimuli on the left upper and lower extremity. Patient had a Glasgow Coma Scale score of 6, NIH stroke scale score of 23, and an Intracerebral Hemorrhage Score of 5. A repeat non-contrast CT scan of the head was performed at 17:00 hours to 
monitor for expansion of hematoma and progression of secondary hydrocephalus. Imaging revealed an interval increase in the size of the acute intraparenchymal hematoma, measuring $4.3 \times 5.2 \times 4.8 \mathrm{~cm}$. In addition, there was an increase in amount of intraventricular hemorrhage, progression of hydrocephalus, and worsening vasogenic edema causing a mass effect with a 5 $\mathrm{mm}$ left midline shift (Figure 1B). At the request of the patient's family members, her code status changed to DNR and she was made comfort care. No interventions were pursued and patient entered hospice care.

Intracerebral hemorrhage (ICH) occurs in about 15\% of strokes per year (1). The most common cause of spontaneous $\mathrm{ICH}$ is rupture of micro-aneurysms of small blood vessels in brain tissue, secondary to chronic hypertension. Hypertensive hemorrhages typically occur in the basal ganglia and thalamus, which are in close proximity to the cerebral ventricular system. Blood can accumulate at these sites forming an acute intraparenchymal hematoma, which can expand and exert mechanical pressure on the ventricular walls leading to intraventricular rupture and secondary intraventricular hemorrhage (IVH) (2). Intraventricular rupture occurs in approximately $45 \%$ of spontaneous $\mathrm{ICH}$, which results in an expected mortality of $50-80 \%$ (1). Blood in the ventricular system can clot forming a "cast" (Figure 1A, red arrow). Ventricular casts are especially troublesome because the cast can block the outflow of cerebrospinal fluid causing an acute obstructive hydrocephalus, which can lead to increased intracerebral pressure (ICP), mass effect, and brain herniation (2). In Figure 1A, the intraventricular cast formation likely represents the patient's normal ventricular size prior to ventriculomegaly from hydrocephalus. Figure 1B shows the typical progression of the intraparenchymal hematoma and obstructive hydrocephalus. There are several treatment options for management of an intraparenchymal hematoma with intraventricular rupture; they include reduction of ICP via ventriculostomy and medical therapy, surgical evacuation of the hematoma, and intraventricular thrombolytics to reduce casting and secondary obstructive hydrocephalus $(2,3)$. Despite these interventions, the prognosis remains poor (3).

Melvin Parasram MS OMS4, ${ }^{1}$ Mangala Gopal OMS4, ${ }^{2}$ Lee Raube DO MS, ${ }^{3}$ Editha Johnson DO, ${ }^{4}$ Deepak Nair MD ${ }^{4,5}$

${ }^{1}$ Midwestern University, Arizona College of Osteopathic Medicine, Glendale, AZ USA

${ }^{2}$ Des Moines University, College of Osteopathic Medicine, Des Moines, IA USA

${ }^{3}$ Departments of Emergency Medicine and ${ }^{4}$ Neurology, University of Illinois College of Medicine at Peoria, Peoria, IL USA

${ }^{5}$ Illinois Neurological Institute, OSF St. Francis Medical Center, Peoria, IL USA

\section{References}

1. Hinson HE, Hanley DF, Ziai WC. Management of intraventricular hemorrhage. Curr Neurol Neurosci Rep. 2010 Mar;10(2):73-82. [CrossRef] [PubMed] 
2. Hanley DF. Intraventricular hemorrhage: severity factor and treatment target in spontaneous intracerebral hemorrhage. Stroke. 2009 Apr;40(4):1533-8. [CrossRef] [PubMed]

3. Nieuwkamp DJ, de Gans K, Rinkel GJ, Algra A. Treatment and outcome of severe intraventricular extension in patients with subarachnoid or intracerebral hemorrhage: a systematic review of the literature. J Neurol. 2000 Feb;247(2):117-21. [CrossRef] [PubMed] 